\title{
ERGODIC MEASURE PRESERVING TRANSFORMATIONS WITH QUASI-DISCRETE SPECTRUM
}

\author{
BY
}

\author{
JAMES B. ROBERTSON( ${ }^{1}$ )
}

\begin{abstract}
It is shown that an ergodic measure preserving transformation with quasi-discrete spectrum is conjugate to: (a) the skew-product of an ergodic measure preserving transformation with discrete spectrum and a measurable family of totally ergodic measure preserving transformations with quasi-discrete spectrum; (b) a factor of the direct product of an ergodic measure preserving transformation with discrete spectrum and a totally ergodic measure preserving transformation with quasi-discrete spectrum. Sufficient conditions are given to insure that an ergodic measure preserving transformation with quasidiscrete spectrum is conjugate to the direct product of an ergodic measure preserving transformation with discrete spec trum and a totally ergodic measure preserving transformation with quasi-discrete spectrum.
\end{abstract}

0. Introduction. The structure of ergodic measure preserving transformations with discrete spectrum and totally ergodic measure preserving transformations with quasi-discrete spectrum has been well studied (cf. Halmos [4, pp. 46-50], Abramov [1], and Hahn and Parry [2]). The purpose of this paper is to reduce as much as possible the study of ergodic measure preserving transformations with quasi-discrete spectrum that are not totally ergodic to the above two cases.

We shall show (Theorem 4.4) that an ergodic measure preserving transformation $T$ with quasi-discrete spectrum is conjugate to a skew product $S \times\left\{S_{x}\right\}$ where $S$ has discrete spectrum and where $S_{x}$ is totally ergodic with quasi-discrete spectrum and whose quasi-eigenfunctions are independent of $x$. Next (Theorem 4.5) if the torsion subgroup of the group of eigenvalues of $T$ is the direct product of a complete group and a group of bounded order, then $T$ is conjugate to the direct product $S_{1} \times S_{2}$ where $S_{1}$ has discrete spectrum and $S_{2}$ is totally ergodic with discrete spectrum. An example $(\$ 5)$ is given to show that if the torsion subgroup of eigenvalues of $T$ is not the direct product of a complete group and a group of bounded order, then in general a factorization as in Theorem 4.5 is not possible. Finally using the above results it is shown (Theorem 4.6) that an ergodic measure preserving transformation with quasi-discrete spectrum is conjugate to a factor of the direct product of a ergodic transformation with discrete spectrum and a

Received by the editors April 23, 1973.

AMS (MOS) subject classifications (1970). Primary 28A65; Secondary 22D40.

Key words and phrases. Measure preserving, totally ergodic, quasiodiscrete spectrum, quasi-eigenvalues.

(1) This research was partially supported by NSF grant GP-19660 while the author was visiting the University of Warwick. 
totally ergodic transformation with quasi-discrete spectrum. It also follows from our results that an ergodic measure preserving transformation with quasi-discrete spectrum whose eigenvalues are all roots of unity has, in fact, discrete spectrum.

1. Definitions and notation. Let $T$ be an ergodic measure preserving transformation acting on a probability space $(\Omega, \mathcal{F}, P)$. We shall denote the group of all complex numbers with absolute value 1 by $K$. If $\mathcal{F}^{\prime}$ is an invariant sub- $\sigma$ algebra of $\mathcal{F}$ (i.e. $T^{-1}\left(\mathcal{F}^{\prime}\right) \subseteq \mathcal{F}^{\prime} \subseteq \mathcal{F}$ ), $\left\langle\mathcal{F}^{\prime}, K\right\rangle$ will denote the group of all $\mathcal{F}^{\prime}$. measurable functions from $\Omega$ to $\bar{K}$. (In $\left\langle\mathcal{F}^{\prime}, K\right\rangle$ we shall identify functions that are equal almost everywhere and the restriction of $P$ to $\mathcal{F}^{\prime}$ will still be denoted by $\left.P_{\text {. }}\right) \mathrm{On}_{\mathrm{n}}\langle\mathcal{F}, K\rangle$ the homomorphism $R$ is defined by $R(f)=(f \circ T) \cdot \bar{f}$. That $T$ is ergodic means that the kernel of $\dot{R}$ is $K$.

The quasi-eigenfunctions of $T$ with respect to $\mathcal{F}^{\prime}$ are defined by $G_{n}\left(T, \mathcal{F}^{\prime}\right)=$ $\left\{f \in\left\langle\mathcal{F}^{\prime}, K\right\rangle: R^{n+1}(f)=1\right\}$ and $G\left(T, \mathcal{F}^{\prime}\right)=\bigcup_{n=0}^{\infty} G_{n}\left(T, \mathcal{F}^{\prime}\right)$. The quasi-eigenvalues are defined by $H_{n}\left(T, \mathcal{F}^{\prime}\right)=R\left[G_{n}\left(T, \mathcal{F}^{\prime}\right)\right]$ and $H\left(T, \mathcal{F}^{\prime}\right)=R\left[G\left(T, \mathcal{F}^{\prime}\right)\right]$. The elements of $G_{1}\left(T, \stackrel{n}{\mathfrak{F}}^{\prime}\right)$ and $H_{1}\left(T, \stackrel{n}{\mathfrak{F}}^{\prime}\right)$ are called eigenfunctions and eigenvalues respectively.

$T$ is said to have quasi-discrete spectrum (resp. discrete spectrum) with respect to $\mathcal{F}^{\prime}$ if $L_{2}\left(\Omega, \mathcal{F}^{\prime}, P\right)=\overline{L\left[G\left(T, \mathcal{F}^{\prime}\right)\right]}$ (resp. $\overline{L\left[G\left(T, \mathcal{F}^{\prime}\right)\right]}$ ) where $L[A]$ denotes the linear space spanned by $A$ and $\overline{L[A]}$ denotes the closure of $L[A]$ in $L_{2}\left(\Omega, \mathcal{F}^{\prime}, P\right)$.

Measure preserving transformations $T$ on $(\Omega, \mathcal{F}, P)$ and $T^{\prime}$ on $\left(\Omega^{\prime}, \mathcal{F}^{\prime}, P^{\prime}\right)$ are said to be conjugate if they induce isomorphic automorphisms on their induced measure algebras (cf. Halmos [4, pp. 42-45]).

Finally we say that a group $C$ is the direct product of groups $A$ and $B$, written $C=A \times B$, if $C=A \cdot B$ and $A \cap B=\{1\}$.

2. The quasi-eigenvalues of $Z_{m}$. Let $m$ be a positive integer. $Z_{m}$ will denote the dynamical system $(\Omega, \mathcal{F}, P, T)$ where $\Omega=\{0, \cdots, m-1\}, \mathcal{F}=2^{\mathfrak{Q}}, P(A)=$ $|A| / m$ (where $|A|$ denotes the number of points in $A$ ), and $T(\omega)=\omega+1(\bmod m)$. We shall set $G(T, \mathcal{F})=G\left(Z_{m}\right)$, etc., for simplicity. We may represent $\langle\mathcal{F}, K\rangle$ as $K^{m}=\left\{\mathbf{x}=\left(x_{1}, \cdots, x_{m}\right): x_{i} \in K, 1 \leq i \leq m\right\}$. Then $R$ is given by $\mathbf{y}=R(\mathbf{x})$ where $y_{i}=x_{i+1} \bar{x}_{i}$ (with $\left.x_{m+1}=x_{1}\right)$. Thus it follows that the range of $R$ is contained in $\mathcal{X}=\left\{\mathrm{x}=\left(x_{1}, \cdots, x_{m}\right) \in K^{m}: \Pi_{i=1}^{m} x_{i}=1\right\}$.

\subsection{Lemma. $R(X)=X$.}

Proof. Clearly $R(\mathscr{X}) \subseteq \mathfrak{X}$ by what was said above. Let $\mathrm{y}=\left(y_{1}, \cdots, y_{m}\right) \in \mathcal{X}$. Define $x_{2}=y_{1} x_{1}, x_{3}=y_{1} y_{2} x_{1}, \cdots, x_{n}=y_{1} \cdots y_{m-1} x_{1}$ and take $x_{1}$ so that $\Pi_{i=1}^{m} x_{i}=x_{1}^{m} y_{1}^{m-1} \cdots y_{m-1}=1$. Then $\mathrm{x}=\left(x_{1}, \cdots, x_{m}\right) \in \mathcal{X}, \bar{x}_{i} x_{i+1}=\bar{y}_{1} \cdots$ $\bar{y}_{i-1} \bar{x}_{1} y_{1} \cdots y_{i} x_{1}=y_{i}$ if $i<m$, and $\bar{x}_{m} x_{1}=\bar{y}_{1} \cdots \bar{y}_{m-1} \bar{x}_{1} x_{1}=y_{m}$ since 
$\mathrm{y} \in \mathcal{X}$. Thus $R(\mathbf{x})=\mathbf{y}$, and hence $\mathfrak{X} \subseteq R(\mathscr{X})$.

\subsection{Lemma. $R\left[H\left(Z_{m}\right)\right]=H\left(Z_{m}\right)$.}

Proof. Clearly $R\left[H\left(T, \mathcal{F}^{\prime}\right)\right] \subseteq H\left(T, \mathcal{F}^{\prime}\right)$ for any transformation. Let $f \epsilon$ $H\left(Z_{m}\right)$. Since $f \in R\left[G\left(Z_{m}\right)\right], f \in \mathcal{X}^{-}$. By Lemma 2.1 there exist $f^{\prime}$ and $f^{\prime \prime} \in \mathcal{X}$ such that $R\left(f^{\prime \prime}\right)=f^{\prime}$ and $R\left(f^{\prime}\right)=f$. Hence $f^{\prime \prime} \in G\left(Z_{m}\right), f^{\prime}=R\left(f^{\prime \prime}\right) \in H\left(Z_{m}\right)$, and $f=R\left(f^{\prime}\right) \in R\left[H\left(Z_{m}\right)\right]$.

Since $K$ is a complete group (i.e. for every $x \in K$ and for every $n>0$ there exists $y \in K$ such that $\left.y^{n}=x\right)$, the exact sequence $1 \rightarrow K \rightarrow G\left(Z_{m}\right) \stackrel{R}{\rightarrow} H\left(Z_{m}\right) \rightarrow 1$ splits, i.e. there is a homomorphism $Q: H\left(Z_{m}\right) \rightarrow G\left(Z_{m}\right)$ such that $R Q$ equals the identity on $H\left(Z_{m}\right)$.

2.3. Lemma. $Q\left[H_{1}\left(Z_{m}\right)\right]$ is an orthonormal basis for $L_{2}\left(Z_{m}\right)$.

Proof. The vectors in $Q\left[H_{1}\left(Z_{m}\right)\right]$ are orthogonal since they are eigenvectors of the unitary operator $\left(x_{1}, \cdots, x_{m}\right) \rightarrow\left(x_{2}, \cdots, x_{m}, x_{1}\right)$ corresponding to distinct eigenvalues. They are normal since their modulus is identically 1 , and they are a basis since there are $m$ of them $\left(H_{1}\left(Z_{m}\right)=\{\exp (2 \pi i k / m): 1 \leq k \leq m\}\right)$ and $L_{2}\left(Z_{m}\right)$ is m-dimensional.

2.4. Lemma. Let $m=m_{1} \cdots m_{k}$ with $m_{i}=p_{i}{ }^{n_{i}}$ and $1<p_{1}<\cdots<p_{k}$ be the prime decomposition of $m$. Then $H\left(Z_{m}\right)$ is isomorpbic to the direct product $H\left(Z_{m_{1}}\right) \times \cdots \times H\left(Z_{m_{k}}\right)$.

Proof. Let $T$ be the direct product of the dynamical systems $Z_{m_{i}}$. Then $T^{j}\left(x_{1}, \cdots, x_{k}\right)=\left(x_{1}+j\left(\bmod m_{1}\right), \cdots, x_{k}+j\left(\bmod m_{k}\right)\right)$. Since $m$ is the least common multiple of $\left\{m_{1}, \cdots, m_{k}\right\}$, the points $\left\{T^{j}\left(x_{1}, \cdots, x_{k}\right): 1 \leq j \leq m\right\}$ are distinct. Hence $T$ is isomorphic to $Z_{m}$. Clearly $H_{n}\left(Z_{m_{1}}\right) \times \cdots \times H_{n}\left(Z_{m_{k}}\right) \subseteq$ $H_{n}(T)$, and $H_{n}\left(Z_{m_{i}}\right) \cap H_{n}\left(Z_{m_{j}}\right)=\{1\}$ since $m_{i}$ and $m_{j}$ are relatively prime if $i \neq j$. It follows from Lemma 2.2 that $\left|H_{n}\left(Z_{m}\right)\right|=m^{n}$. Thus $\mid H_{n}\left(Z_{m_{1}}\right) \times \cdots$ $\times H_{n}\left(Z_{m_{k}}\right)\left|=\Pi_{j=1}^{k} m_{j}^{n}=m^{n}=\right| H_{n}(T) \mid$ and hence $H_{n}(T)=H_{n}\left(Z_{m_{1}}\right) \times \cdots$ $\times H_{n}\left(Z_{m_{k}}\right)$. 口

We shall need the following result from number theory.

2.5. Lemma. Let $p$ be a prime and $n$ a positive integer. Then $p$ divides $a_{k}=(-1)^{k}\left(\begin{array}{c}p^{n}-1 \\ k\end{array}\right)-1$ for all $k$ between 0 and $p^{n}-1$.

Proof by induction on $k$. Since $a_{0}=1-1=0, p$ divides $a_{0}$. Suppose $p$ divides $a_{k-1}$ and $k \leq p^{n}-1$. Then

$$
a_{k}=(-1)^{k} p^{n}\left(\begin{array}{c}
p^{n}-1 \\
k-1
\end{array}\right) / k+a_{k-1} .
$$


By induction $p$ divides $a_{k-1}$ and since $p^{n}\left(\begin{array}{c}p^{n}-1 \\ k-1\end{array}\right) / k$ is an integer, $k$ divides $p^{n}\left(p^{n}-1\right)$. Since $k<p$ and $p$ is a prime, $p \cdot k$ divides $p^{n}\left(\begin{array}{c}p^{n}-1 \\ k-1\end{array}\right)$. Thus $p$ divides $p^{n}\left(\begin{array}{c}k=1 \\ k=1\end{array}\right) / k$ and hence $p$ divides $a_{k}$.

2.6. Lemma. Let $p$ be a prime number, let $n$ be a positive integer and $m=$ $p^{n}$. Then $H\left(Z_{m}\right)$ is the set of all $\mathrm{x} \in \mathcal{X}$ sucb that there exists a positive $k$ with $\mathbf{x}^{p^{k}}=1$. Hence $H\left(Z_{m}\right)$ is isomorphic to the direct product of $m-1$ copies of the complete group $p^{\infty}=\left\{\exp \left(2 \pi i r / p^{s}\right): r, s \geq 0\right\}$.

Proof. Let $\mathbb{R}=\left\{x \in \mathcal{X}: \mathrm{x}^{p^{k}}=1\right.$ for some $\left.k>0\right\}$. By Lemma 2.2, $H\left(Z_{m}\right)=$ $\left\{\mathrm{x} \in \mathcal{X}: R^{k}(\mathrm{x})=1\right.$ for some $\left.k>0\right\}$. Suppose $R(\mathrm{x})=\mathrm{y}$ with $\mathrm{x} \in \mathcal{X}$ and $\mathrm{y} \in \mathbb{m}$ (say. $\left.\mathrm{y}^{p^{l}}=1\right)$. Then $R\left(\mathrm{x}^{p^{l}}\right)=[R(\mathrm{x})]^{p^{l}}=\mathrm{y}^{p^{l}}=1$. Therefore $\mathrm{x}^{p^{l}}=\exp \left(2 \pi i k / p^{n}\right) \cdot 1$ for some $k$, and hence $x^{p(l+n)}=1$. Thus $x \in \pi$. Since $l \in \pi$, it follows that $H\left(Z_{m}\right) \subseteq \Re$.

Now suppose that $\mathrm{x} \in \mathbb{M}$ with $\mathrm{x}^{p^{l}}=1$. Let $\mathrm{y}=R^{k}(\mathrm{x})$. Then $y_{j}=$ $\Pi_{i=0}^{k}\left[x_{j+i}\right]^{a}$ where $a=(-1)^{k+i}\left({ }_{i}^{k}\right)$, as can be seen by induction with $j+i$ being interpreted $\bmod m$. Letting $k=m-1$ we have

$$
y_{j}=\prod_{i=0}^{m-1}\left[x_{j+i}\right]^{b} \text { where } b_{i}=(-1)^{m-1+i}\left(\begin{array}{c}
m-1 \\
i
\end{array}\right) .
$$

Since $\mathrm{x} \in \mathcal{X}, x_{j+m-1}=\Pi_{i=0}^{m-2} \bar{x}_{j+i}$. Therefore

$$
y_{j}=\prod_{i=0}^{m-2}\left[x_{j+i}\right]^{c} i \text { where } c_{i}=(-1)^{m-1+i}\left(\begin{array}{c}
m-1 \\
i
\end{array}\right)-1 .
$$

Thus if $p \neq 2, y_{j}=\Pi_{i=0}^{m-2}\left[x_{j+i}\right]^{a_{i}}$. Since, by Lemma 2.5, $p$ divides $a_{i}$, we have

$$
y_{j}^{p l-1}=\prod_{i=0}^{m-2}\left[x_{j+i}\right]^{l-1} a_{i}=1 \text {. }
$$

Hence $\left[R^{m-1}(\mathrm{x})\right]^{p^{l-1}}=1$. Thus, by induction, $R^{r}(\mathrm{x})=1$ for $r=\Sigma_{1}^{l} p^{i}-l$. Hence $x \in H\left(Z_{m}\right)$ which implies $\mathbb{N} \subseteq H\left(Z_{m}\right)$.

If $p=2$, (1) becomes $y_{j}=\Pi_{i=0}^{m-2}\left[x_{j+i}\right]^{-a_{i}-2}$, and since 2 divides $-a_{i}-2$, the proof proceeds as above.

\subsection{Lemma. Let $m>0$. Then $H\left(Z_{m}\right)$ is a complete torsion group.}

Proof. By Lemma 2.4, $H\left(Z_{m}\right)$ is the direct product of groups isomorphic to $H\left(Z_{p} k\right)$ where $p$ is a prime. By Lemma 2.6 , therefore, it is isomorphic to the direct product of groups $p^{\infty}$ where $p$ is a prime. Since these are complete torsion groups, so is $H\left(Z_{m}\right)$.

3. The quasi-eigenvalues of $T$. Let $T$ be an ergodic measure preserving transformation acting on a probability space $(\Omega, \mathcal{F}, P)$. Throughout this section 
quasi-eigenfunction, etc., will be with respect to $\mathcal{F}^{\prime}$ and we will write $G=$ $G\left(T, \mathcal{F}^{\prime}\right)$, etc., for simplicity. If $A$ is a group, $\tilde{A}=\left\{a \in A: a^{n}=1\right.$ for some $n>$ $0\}$ will denote the torsion subgroup of $A$.
3.1. Lemma.
(a) $R[\tilde{H}]=\tilde{H}$.
(b) $\tilde{H}$ is a complete group.
(c) $\tilde{H} \subseteq L\left[G_{1}\right]$.

Proof. Since $R$ is a homomorphism and $R[H] \subseteq H$, we have $R[\tilde{H}] \subseteq \tilde{H}$. Let

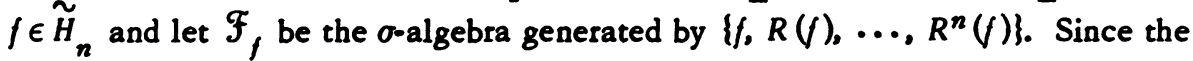
algebra of functions generated by $\left\{f, R(f), \ldots, R^{n}(f)\right\}$ is invariant under composition with $T$ and since each $R^{k}(f)$, being a root of unity, assumes only finitely many different values, it follows that $\mathcal{F}_{f}$ is a finite invariant $\sigma$-algebra. Since $T$ is ergodic, it follows that $Z_{n}$ is a factor of $T$ where $n$ is the number of atoms in $\mathcal{F}_{f}$, i.e. there is a measure preserving transformation $\phi: \Omega \rightarrow Z_{n}$ such that $\phi^{-1}\left(2^{Z} n\right)=\mathcal{F}_{f}$. There is therefore a quasi-eigenvalue $g$ in $H\left(Z_{n}\right)$ such that $f=g^{\circ} \phi$. It follows from Lemmas 2.2 and 2.7 that for every $n>0$ there exist quasi-eigenvalues $g^{\prime}$ and $g^{\prime \prime}$ such that $R\left(g^{\prime}\right)=g$ and $g^{\prime \prime}=g$. Setting $f^{\prime}=$ $g^{\prime} \circ \phi$ and $f^{\prime \prime}=g^{\prime \prime} \circ \phi$, we see that $f^{\prime}, f^{\prime \prime} \in \tilde{H}, R\left(f^{\prime}\right)=f$, and $f^{\prime \prime n}=f$. Thus $\tilde{H}$ is complete and $R[\tilde{H}]=\widetilde{H}$. Since, by Lemma 2.3,g $\in L\left[G_{1}\left(Z_{n}\right)\right]$, it follows that $\tilde{H} \subseteq L\left[G_{1}\right]$

3.2. Lemma. There exists a sequence of groups $H_{n}^{*}$ sucb that (a) $H_{n}^{*} \subseteq$ $H_{n+1}^{*}$; (b) $\tilde{H} \cdot H_{n}=\tilde{H} \times H_{n}^{*}$.

Proof. Let $L_{n}=\tilde{H} \cdot H_{n}$. Then $\tilde{L}_{n}=\tilde{H}$. Since $\tilde{H}$ is complete, there exists a group $H_{\mathcal{L}}^{*}$ such that $L_{1}=\widetilde{H} \times H_{1}^{*}$. Proceeding by induction $\tilde{H} \times H_{n_{-1}}^{*} \subseteq L_{n}$, and $\tilde{L}_{n}=\overparen{H}$. Thus there exists $H_{n}^{*}$ such that $H_{n-1}^{*} \subseteq H_{n}^{*}$ and $L_{n}=\widetilde{H} \times \bar{H}_{n}^{*}$.

The group $H^{*}=\bigcup_{n=1}^{\infty} H_{n}^{*}$ in general will not be invariant under $R$. The remaining lemmas of this section will establish sufficient conditions for the existence of an invariant $H^{*}$.

3.3. Lemma. $H_{n} /\left[H_{n-1} \cdot \widetilde{H}_{n}\right]$ is torsion free.

Proof by induction on $n$. The case $n=1, H_{1} / \tilde{H}_{1}$, is immediate. Suppose the lemma is true for $n \geq 1$ and let $f \in H_{n+1} \sim_{\text {with }} f^{m} \in H_{n} \cdot \tilde{H}_{n+1}$. Let $g=$ $R(f)$. Then $g \in H_{n}$ and $g^{m}=R\left(f^{m}\right) \in H_{n-1}^{n+1} \cdot \tilde{H}_{n}$. Thus by induction $g=g^{\prime} \cdot g^{\prime \prime} \epsilon$ $H_{n-1} \cdot \tilde{H}_{n}$. By Lemma 3.1(a) there exists $f^{\prime \prime} \in \tilde{H}_{n+1}$ with $R\left(f^{\prime \prime}\right)=g^{n}$. Let $f^{\prime}=f \cdot f^{\prime \prime}$. Since $R\left(f^{\prime}\right)=g \cdot \overline{g^{\prime \prime}}=g^{\prime} \in H_{n-1}, f^{\prime} \in H_{n^{\circ}}$. Thus $f=f^{\prime} \cdot f^{\prime \prime} \in H_{n}$. $\tilde{H}_{n+1}$ which completes the proof.

Next we need a group theoretic lemma.

3.4. Lemma. Let $\boldsymbol{G}$ be an abelian group whose torsion subgroup $\tilde{G}$ is the direct product of a complete group and a group of bounded order. Let $H$ be a 
subgroup of $G$ such that $\tilde{G} \cap H=\{1\}$. If $G /[\tilde{G} \times H]$ is torsion free, then there exists a subgroup $H^{*}$ such that $H \subseteq H^{*}$ and $G=\widetilde{G} \times H^{*}$.

Proof. First note that the lemma, in the case $H=\{1\}$, is a standard result (cf., e.g. [5, p. 202]). Let $\pi: G \rightarrow G / H$ be the natural projection and suppose $\pi(g) \in G / H$, i.e. there exists a positive $n$ such that $g^{n} \in H \subseteq \tilde{G} \times H$. Since $G /[\tilde{G} \times H]$ is torsion free, $g=\tilde{g} \cdot g^{\prime} \in \tilde{G} \times H$. Thus $\pi(\tilde{g})=\pi(g)$. Thus we have shown that $\pi[\tilde{G}]=\overparen{G / H}$. Since $\tilde{G} \cap H=\{1\}, \pi \mid \widetilde{G}$ is an isomorphism onto $\overparen{G / H}$. Thus by the first part there exists a subgroup $H^{\prime} \subseteq G / H$ such that $G / H=$ $(G / H) \times H^{\prime}$. It is easy to verify that $H^{*}=\pi^{-1}\left(H^{\prime}\right)$ satisfies the conditions of the lemma.

3.5. Lemma. If $\tilde{H}_{1}$ is the direct product of a complete group and a group of bounded order, then there exist groups $H_{n}^{*}$ such that

(a) $H_{n-1}^{*} \subseteq H_{n}^{*}$;

(b) $H_{n}=\tilde{H}_{n} \times H_{n}^{*}$

(c) $R\left[H_{n}^{*}\right] \subseteq H_{n-1}^{*}$.

Proof by induction on $n$. By Lemma 3.4 there exists a group $H_{1}^{*}$ such that $H_{1}=\widetilde{H}_{1} \times H_{1}^{*}$. Setting $H_{0}^{*}=\{1\}$ we have $(\mathrm{a})-(\mathrm{c})$ for $n=1$. Suppose now that we have constructed $H_{0}^{*}, \cdots, H_{n}^{*}$. Let $L=H_{n+1} \cap R^{-1}\left[H_{n}^{*}\right]$. If $f \in \tilde{L}$, then $R(f) \in \tilde{H} \cap H_{n}^{*}=\{1\}$. Hence $\widetilde{L}=\tilde{H}_{1}$. Suppose $f \in L \subseteq{ }_{\sim}{ }_{n+1}$ and $f^{m} \in \widetilde{L} \times H_{n}^{*} \subseteq$ $H_{n} \cdot \tilde{H}_{n+1}$. By Lemma 3.3 there exist $f^{\prime} \in H_{n}$ and $\tilde{f} \in \tilde{\tilde{H}}_{n+1}$ such that $f=$

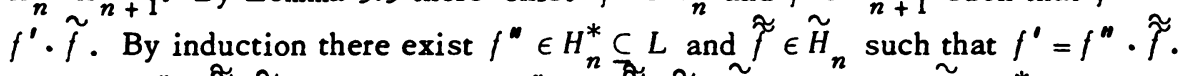
Thus $f=f^{\prime \prime} \cdot(\tilde{f} \cdot \tilde{f})$. Since $f$ and $f^{\prime \prime} \in \tilde{L}, \tilde{f} \cdot \tilde{f} \in \tilde{L}$. Hence $f \in \tilde{L} \times H_{n}^{*}$. Thus $L /\left[\tilde{L} \times H_{n}^{*}\right]$ is torsion free, and hence by Lemma 3.4 there exists $H_{n+1}^{*}$ such that $L=\widetilde{L} \times H_{n+1}^{*}$ and $H_{n}^{*} \subseteq H_{n+1}^{*}$. If $f \in H_{n+1}$, then $R(f)=\tilde{g} \cdot g^{*}$ where $\tilde{\boldsymbol{g}} \in \widetilde{H}_{n}$ and $\boldsymbol{g}^{*} \in H_{n}^{*}$. By Lemma 3.1 there exists $\tilde{f} \in H_{n+1}^{*}$ such that $R(\tilde{f})=$ $\tilde{g}$. Let $f^{\prime}=f \cdot \overline{\widetilde{f}}$. It follows that $f^{\prime} \in L$ and hence $f^{\prime}=f^{*} \cdot \tilde{f}$ where $f^{*} \in H_{n+1}^{*}$ and $\tilde{f} \in \tilde{H}_{1}$. Hence $H_{n+1} \subseteq \tilde{H}_{n+1} \cdot H_{n+1}^{*}$. Clearly $H_{n+1}^{*} \cap \tilde{H}_{n+1}=\{1\}$ and $R\left[H_{n+1}^{*}\right] \subseteq R[L] \subseteq H_{n}^{*}$ by definition.

4. The quasi-eigenfunctions of $T$. Since $K$ is a complete group, the exact sequence $1 \rightarrow K \rightarrow G\left(T, \mathcal{F}^{\prime}\right) \stackrel{R}{\rightarrow} H\left(T, \mathcal{F}^{\prime}\right) \rightarrow 1$ splits, i.e. there exists a homomorphism $Q: H\left(T, \mathcal{F}^{\prime}\right) \rightarrow G\left(T, \mathcal{F}^{\prime}\right)$ such that $R Q$ is the identity on $H\left(T, \mathcal{F}^{\prime}\right)$. Throughout this section $Q$ will be such a homomorphism. We shall also suppose that we have a decomposition $H\left(T, \mathcal{F}^{\prime}\right)=\widetilde{H}\left(T, \mathcal{F}^{\prime}\right) \times H^{*}\left(T, \mathcal{F}^{\prime}\right)$ as in Lemma 3.2 and will set $\Phi_{n}\left(T, \mathcal{F}^{\prime}\right)=Q\left[H_{n}\left(T, \mathcal{F}^{\prime}\right)\right], \tilde{\Phi}_{n}\left(T, \mathcal{F}^{\prime}\right)=Q\left[\tilde{H}_{n}\left(T, \mathcal{F}^{\prime}\right)\right]$, etc.

4.1. Lemma. If $f \in \Phi_{n}\left(T, \mathcal{F}^{\prime}\right)$, then either $f \in \tilde{\Phi}\left(T, \mathcal{F}^{\prime}\right)$ or $f \perp \tilde{\Phi}\left(T, \mathcal{F}^{\prime}\right)$. 
Proof by induction on $n$. If $f \in \Phi_{1}\left(T, \mathcal{F}^{\prime}\right)$ and $f \notin \widetilde{\Phi}\left(T, \mathcal{F}^{\prime}\right)$, then $f \perp \tilde{\Phi}_{1}(T, \mathcal{F})$ since eigenfunctions corresponding to distinct eigenvalues are orthogonal. Thus, by Lemma $3.1, f \perp \overline{L\left[\widetilde{\Phi}_{1}\left(T, \mathcal{F}^{\prime}\right)\right]} \supseteq \widetilde{\Phi}\left(T, \mathcal{F}^{\prime}\right)$. Suppose therefore that the lemma is true for $\Phi_{n}\left(T, \mathcal{F}^{\prime}\right)$, that $f \in \Phi_{n+1}\left(T, \mathcal{F}^{\prime}\right)$, and that $f \notin \widetilde{\Phi}\left(T, \mathcal{F}^{\prime}\right)$. As above it remains to prove that $f 1 \widetilde{\Phi}_{1}\left(T, \tilde{F}^{\prime}\right)$. Let $k$ be the smallest integer such that $R^{k}(f) \in \tilde{\Phi}\left(T, \mathcal{F}^{\prime}\right)$. Suppose first that $k \neq n+2$. By Lemma 3.1 there exists $\tilde{f} \in \tilde{\Phi}\left(T, \mathcal{F}^{\prime}\right)$ such that $R^{k}(\tilde{f})=R^{k}(f)$. Let $f^{\prime}=f \cdot \tilde{f}$. Since $R^{k}\left(f^{\prime}\right)=1, f^{\prime} \epsilon$ $\Phi_{k-1}\left(T, \mathcal{F}^{\prime}\right)$ and $f^{\prime} \notin \widetilde{\Phi}\left(T, \mathcal{F}^{\prime}\right)$. Therefore by induction $f^{\prime} \perp \tilde{\Phi}\left(T, \mathcal{F}^{\prime}\right)$. Hence $f=f^{\prime} \cdot \tilde{f} \perp \tilde{\Phi}\left(T, \mathcal{F}^{\prime}\right)$.

Suppose therefore that $\lambda=R^{n+1}(f)$ is not a root of unity $\left(R^{n+2}(f)=1 \epsilon\right.$ $\tilde{\Phi}\left(T, \mathcal{F}^{\prime}\right)$ ). If $\tilde{f} \in \widetilde{\Phi}_{1}\left(T, \mathcal{F}^{\prime}\right)$, then $\lambda=R^{n+1}(b)$ where $b=f \cdot \widetilde{f}$. Following Hahn and Parry [2, Theorem 1] we have

$$
b \circ T^{k}=\prod_{j=0}^{n+1}\left[R^{j}(b)\right]^{\left(\begin{array}{c}
k \\
j
\end{array}\right)}=\exp \left(2 \pi i \sum_{j=0}^{n+1}\left(\begin{array}{l}
k \\
j
\end{array}\right) a_{j}(\cdot)\right)=\exp (2 \pi i p(k))
$$

where $R^{j}(b)=\exp \left(2 \pi i a_{j}().\right)$. Then $p($.$) is a polynomial of degree n+1$ with leading coefficient $a_{n+1}$ which is irrational since $\lambda=\exp \left(2 \pi i a_{n+1}\right)$ is not a root of unity. Thus Weyl's theorem (cf. $\left[6\right.$, p. 326]) implies that $(1 / N) \Sigma_{k=1}^{N} b \circ$ $T^{k} \rightarrow 0$ as $N \rightarrow \infty$, and the ergodic theorem implies that $\langle f, \tilde{f}\rangle=E(b)=$ $\lim (1 / N) \Sigma_{k=1}^{N} b \circ T^{k}=0$. Thus $f \perp \widetilde{\Phi}_{1}\left(T, \mathcal{F}^{\prime}\right)$.

4. 2. Lemma. Let $\tilde{F}^{\prime}$ and $\mathcal{F}^{\prime *}$ be the $\sigma$-algebras generated by $\widetilde{\Phi}\left(T, \mathcal{F}^{\prime}\right)$ and $\Phi^{*}(T, \mathcal{F})$ respectively and let $\mathcal{F}^{\prime \prime}=\dddot{F}^{\prime} \vee \mathcal{F}^{\prime *}$. Then $\dddot{F}^{\prime}$ and $\mathcal{F}^{\prime *}$ are independent and $\widetilde{\Phi}_{1}\left(T, \mathcal{F}^{\prime}\right) \times \Phi^{*}\left(T, \mathcal{F}^{\prime}\right)$ is an orthogonal basis for $L_{2}\left(\Omega, \mathcal{F}^{\prime \prime}, P\right)$.

Proof. It follows immediately from Lemma 4.1 that $\Phi^{*}\left(T, \mathcal{F}^{\prime}\right)$ is an orthonormal basis for $L_{2}\left(\Omega, \mathcal{F}^{\prime *}, P\right)$, and from Lemma 3.1 that $\tilde{\Phi}_{1}\left(T, \mathcal{F}^{\prime}\right)$ is an orthonormal basis of $L_{2}\left(\Omega, \tilde{F}^{\prime}, P\right)$. It al so follows from Lemma 4.1 that if $\tilde{f} \in \tilde{\Phi}_{1}\left(T, \mathcal{F}^{\prime}\right)$ and $f^{*} \in \Phi^{*}\left(T, \mathcal{F}^{\prime}\right)$, then $\tilde{f} \perp f^{*}$ unless $\tilde{f}=f^{*}=1$. Thus if $\tilde{A} \in \tilde{F}^{\prime}$ and $A^{*} \in \mathcal{F}^{\prime *}$, using orthogonal expansions we have $P\left(A \cap A^{*}\right)=\left\langle\chi_{\mathbb{A}}, 1\right\rangle\left\langle\chi_{A^{*}}, 1\right\rangle$ $=P(\tilde{A}) \cdot P\left(A^{*}\right)$. Thus the lemma follows.

Since $\widetilde{F}^{\prime}$ and $\mathcal{F}^{\prime *}$ are independent, $T$ on $\left(\Omega, \mathcal{F}^{\prime \prime}, P\right)$ is conjugate to a transformation $T$ on $\left(X, \mathcal{F}_{X}^{\prime}, P_{X}\right) \times\left(Y, \mathcal{F}_{Y}^{\prime}, P_{Y}\right)$ where $\tilde{F}^{\prime}$-measurable functions on $\Omega$ correspond to $\mathcal{F}_{X}^{\prime}$-measurable functions on $X$, and the $\mathcal{F}^{\prime}{ }^{*}$-measurable functions on $\Omega$ correspond to $\mathcal{F}_{Y}^{\prime}$-measurable functions on $Y$. Since $R\left[\tilde{H}\left(T, \mathcal{F}^{\prime}\right)\right]=$ $\tilde{H}\left(T, \mathcal{F}^{\prime}\right), T$ induces a transformation $S$ on $\left(X, \mathcal{F}_{X}^{\prime}, P_{X}\right)$ and a measurable family of transformations $\left\{S_{x}: x \in X\right\}$ on $\left(Y, \mathcal{F}_{Y}^{\prime}, P_{Y}\right)$ such that $T(x, y)=$ $\left(S(x), S_{x}(y)\right)$, i.e. $T$ is the skew product $S_{\times}\left\{S_{x}: x \in X\right\}$. We shall identify $\left(\Omega, \mathcal{F}^{\prime \prime}, P\right)$ with $\left(X, \mathcal{F}_{X}^{\prime}, P_{X}\right) \times\left(Y \times \mathcal{F}_{Y}^{\prime}, P_{Y}\right)$. 
4.3. Lemma. (a) $G\left(S, \mathcal{F}_{X}^{\prime}\right)=K \times \widetilde{\Phi}\left(T, \mathcal{F}^{\prime}\right)$.

(b) $G\left(S_{x^{\prime}}, \mathcal{F}_{Y}^{\prime}\right)=K \times \Phi^{*}\left(T, \mathcal{F}^{\prime}\right)$ for almost all $x$.

(c) For $f \in \Phi_{n}^{*}\left(T, \mathcal{F}^{\prime}\right), f \circ S_{x}(y)=\tilde{g}(x) \cdot g^{*}(y) \cdot f(y)$ where $\tilde{g} \in G\left(S, \mathcal{F}_{X}^{\prime}\right)$ and $g^{*} \in \Phi_{n-1}^{*}\left(T, \mathcal{F}^{\prime}\right)$.

(d) $H_{1}\left(S_{x}, \mathcal{F}_{Y}^{\prime}\right)$ bas no nontrivial roots of unity.

Proof. (a) is immediate and we shall prove (b) - (d) by induction on $n$. Let $f \in \Phi_{1}^{*}\left(T, \mathcal{F}^{\prime}\right)$. Then $R(f)=g \in H_{1}^{*}\left(T, \mathcal{F}^{\prime}\right) \subseteq \tilde{H}\left(T, \mathcal{F}^{\prime}\right) \cdot H_{1}\left(T, \mathcal{F}^{\prime}\right)$. Thus $g=\lambda \tilde{g}$ and if $f \neq 1, \lambda$ will not be a root of unity. Now $f \circ S_{x}(y)=f(T(x, y))=$ $(R(f))(x, y) \cdot f(y)=\lambda \cdot \tilde{g}(x) \cdot f(y)$. Hence $f$ is an eigenvalue of $S_{x}$ with eigenvalue $\lambda \cdot \tilde{g}(x)$. Suppose we have shown that $\Phi_{\eta}^{*}\left(T, \mathcal{F}^{\prime}\right) \subseteq G_{n}\left(S_{x^{\prime}}, \mathcal{F}_{Y}^{\prime}\right)$. Let $f \epsilon$ $\Phi_{n+1}^{*}\left(T, \mathcal{F}^{\prime}\right)$. Then $g=R(f) \in H_{n+1}^{*}\left(T, \mathcal{F}^{\prime}\right) \subseteq \widehat{H}\left(T, \mathcal{F}^{\prime}\right) \cdot H_{n+1}^{n}\left(T, \mathcal{F}^{\prime}\right)$, say $g=$ $\tilde{g} \cdot g^{\prime}$. Therefore $f \circ S_{x}(y)=f(T(x, y))=\tilde{g}(x) \cdot g^{\prime}(x, y) \cdot f(y)$. Now $g^{\prime} \in H_{n+1}\left(T, \mathcal{F}^{\prime}\right)$ $\subseteq K \times \widetilde{\Phi}_{n}\left(T, \mathcal{F}^{\prime}\right) \times \mathscr{\Phi}^{*}\left(T, \mathcal{F}^{\prime}\right)$, say $g^{\prime}(x, y)=\lambda \cdot \widetilde{g}(x) \cdot g^{*}(y)$. Thus $R_{x}(f)(y)=$ $\left(f \circ S_{x}\right) \cdot \tilde{f}=\lambda \tilde{g}(x) \cdot \tilde{g}(x) \cdot g^{*}(y)$. Since $g^{*} \in G_{n}\left(S_{x^{\prime}}, \mathcal{F}_{Y}^{\prime}\right)$ by induction, we have $\Phi_{n+1}^{*}\left(T, \mathcal{F}^{\prime}\right) \subseteq G_{n+1}\left(S_{x^{\prime}}, \mathcal{F}_{Y}^{\prime}\right)$. Since $\tilde{H}_{1}\left(S_{x^{\prime}}, \mathcal{F}_{Y}^{\prime}\right)=\{1\}$, it follows from Lemma 4.1 that $G\left(S_{x^{\prime}}, \mathcal{F}_{Y}^{\prime}\right)=K \times \Phi^{*}\left(S_{x^{\prime}}, \mathcal{F}_{Y}^{\prime}\right)$. Since $\Phi^{*}\left(T, \mathcal{F}^{\prime}\right)$ is an orthonormal basis of $L_{2}\left(Y, \mathcal{F}_{Y}^{\prime}, P_{Y}\right)$, it follows that $G\left(S_{x^{\prime}}, \mathcal{F}_{Y}^{\prime}\right)=K \times \Phi^{*}\left(T, \mathcal{F}^{\prime}\right)$.

A transformation is said to be totally ergodic if $T^{k}$ is ergodic for all $k>0$. For an ergodic transformation this is equivalent to saying that there are no eigenvalues, other than 1 , that are roots of unity. Thus the transformations $S_{x}$ above are totally ergodic.

4.4. Theorem. Let $T$ be an ergodic measure preserving transformation on $(\Omega, \mathfrak{F}, P)$ that has quasi-discrete spectrum with respect to $\mathfrak{F}$. Then $T$ is conjugate to the skew product $S \times\left\{S_{x}: x \in X\right\}$ on $\left(X \times Y, \mathcal{F}_{X} \times \mathcal{F}_{Y}, P\right)$ where $S$ bas discrete spectrum with respect to $\mathcal{F}_{X}$ and $H\left(S, \mathcal{F}_{X}\right)=\widetilde{H}(T, \mathcal{F})$, and where, for each $x, S_{x}$ is totally ergodic with quasi-discrete spectrum with respect to $\mathcal{F}_{Y}$ whose quasi-eigenfunctions are independent of $x$.

The proof follows at once from Lemma 4.5. In [2] Hahn and Parry represent a totally ergodic measure preserving transformation $T$ with quasi-discrete spectrum acting on a Lebesgue space as a minimal affine transformation $T(g)=$ $a \cdot A(g)$ on a compact abelian group. In this case our $S_{x}$ will be given by $S_{x}(g)=$ $a_{x} \cdot A(g)$ where the translation $a_{x}$ (but not the automorphism $A$ ) will depend on $x_{\text {. }}$

4.5. Theorem. Let $T$ be as in Theorem 4.4 and suppose that $\tilde{H}_{1}(T, \mathcal{F})$ is the direct product of a complete group and a group of bounded order. Then $T$ is conjugate to the direct product $S_{1} \times S_{2}$ on $\left(X \times Y, \mathcal{F}_{X} \times \mathcal{F}_{Y}, P\right)$ where $S_{1}$ bas discrete spectrum with respect to $\mathcal{F}_{X}$ and $H\left(S_{1}, \mathcal{F}_{X}\right)=\tilde{H}(T, \mathcal{F})$, and where $S_{2}$ is totally ergodic with quasi-discrete spectrum with respect to $\mathfrak{F}_{\mathbf{Y}}$. 
Proof. By Lemma 3.5 we may choose $H^{*}(T, \mathcal{F})$ such that $R\left[H^{*}(T, \mathcal{F})\right] \subseteq$ $H^{*}(T, \mathcal{F})$. Hence $\mathcal{F}_{Y}=$ the $\sigma$-algebra generated by $Q\left[H^{*}(T, \mathcal{F})\right]$ is an invariant $\sigma$-algebra and $\left\{S_{x}: x \in X\right\}$ on $\left(Y, \mathcal{F}_{Y}, P\right)$ in Theorem 4.4 reduces to $S_{2}$ on $(Y$, $\mathcal{F}_{\boldsymbol{Y}}, P$ ).

The transformation $S_{1}$ on $\left(X, \mathcal{F}_{X}, P_{X}\right)$ is obtained from $T$ on $(\Omega, \mathcal{F}, P)$ in a unique way. However the transformations $S_{2}$ on $\left(Y, \mathcal{F}_{Y}, P_{Y}\right)$ will depend on the factorization $H=\tilde{H} \times H^{*}$. In general there will be many possible such factorizations with different $H_{1}^{*}$. Thus there will be many nonconjugate $S_{2}$ 's to choose from and there appears to be no naturally preferable one.

We shall now show that we can always write the given transformation as a factor of a transformation that is a direct product as in Theorem 4.5.

4.6. Theorem. Let $T$ be an ergodic transformation on $(\Omega, \mathcal{F}, P)$ with quasi-

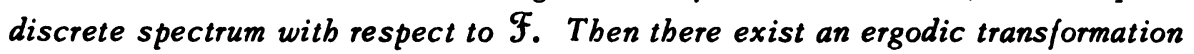
$S_{1}$ on $\left(X, \mathcal{F}_{X}, P_{X}\right)$ with discrete spectrum with respect to $\mathfrak{F}_{X}$, and a totally ergodic transformation $S_{2}$ on $\left(Y, \mathcal{F}_{Y}, P_{Y}\right)$ with quasi-discrete spectrum with respect to $\mathcal{F}_{Y}$, and an invariant subgroup $G^{\prime} \subseteq G\left(S_{1}, \mathcal{F}_{X}\right) \times G\left(S_{2}, \mathcal{F}_{Y}\right)$ such that $T$ is conjugate to $S_{1} \times S_{2}$ on $\left(X \times Y, \mathcal{F}^{\prime}, P_{X} \times P_{Y}\right)$ where $\mathcal{F}^{\prime}$ is the $\sigma$ algebra generated by $G^{\prime}$.

Proof. Let $T$ be written as a skew product $S_{\underset{x}{x}}\left\{S_{x}: x \in X\right\}$ as in Theorem 4.4. Let $L$ be the smallest complete subgroup of $\widetilde{K}$ containing $\widetilde{H}_{1}(T, \mathcal{F})$ and let $S^{\prime}$ be the ergodic transformation on $\left(X^{\prime}, \mathcal{F}_{X}, P_{X}\right)$ with discrete spectrum and eigenvalues $L$ (cf. e.g. Halmos [4, p. 48, Corollary 1]). There is a natural homomorphism $\phi:\left(X^{\prime}, \mathfrak{F}_{X}, P_{X}, S^{\prime}\right) \rightarrow\left(X, \mathcal{F}_{X}, P_{X}, S\right)$. The measurable family of measure preserving transformations $\left\{S_{x}: x \in X\right\}$ may therefore be extended to a measurable family $\left\{S_{x^{\prime}}: x^{\prime} \in X^{\prime}\right\}$ by setting $S_{x^{\prime}}=S_{\phi\left(x^{\prime}\right)}$. Lemma 4.3(c) shows that the elements of $\Phi^{*}(T, \mathcal{F})$ will be quasi-eigenfunctions for the skew product $T^{\prime}=S^{\prime} \times\left\{S_{x^{\prime}}: x^{\prime} \in X^{\prime}\right\}$. Moreover $T^{\prime}$ is ergodic. (For suppose $f$ is a nonconstant invariant function and suppose $\tilde{g} \in \Phi_{1}\left(S^{\prime}, \mathcal{F}_{X}{ }^{\prime}\right)$ and $g^{*} \in \Phi^{*}(T, \mathcal{F})$ with $R(\tilde{g})=\lambda$. We may take $f \perp 1$. Then $\left(f \cdot \tilde{g} \cdot g^{*}\right) \circ T^{\prime k}=$ $f \cdot \tilde{g} \cdot \lambda^{k} \cdot g^{*} \circ T^{k}$. Thus using Weyl's theorem and the ergodic theorem as in the proof of Lemma 4.1 , we find that $E\left(f \cdot \tilde{g} \cdot g^{*}\right)=0$. Hence $f \perp \Phi_{1}\left(S^{\prime}, \mathcal{F}_{X},\right) \times$ $\Phi^{*}(T, \mathcal{F})$, and since these functions generate $L_{2}\left(X^{\prime} \times Y, \mathcal{F}_{X}, \mathcal{F}_{Y}, P_{X}, \times P_{Y}\right)$, $f$ must be zero almost everywhere which is a contradiction.) Therefore $T^{\prime}$ has quasi-discrete spectrum on $\left(X^{\prime} \times Y, \mathcal{F}_{X}, \times \mathcal{F}_{Y}, P_{X}, \times P_{Y}\right)$. Let $\mathcal{F}^{\prime}$ be the invariant $\sigma$-algebra generated by $\Phi_{1}(S, \mathscr{F})_{\times} \Phi^{*}(T, \mathscr{F})$. Then $T$ on $(\Omega, \mathcal{F}, P)$ is clearly conjugate to $T^{\prime}$ on $\left(X^{\prime} \times Y, \mathcal{F}^{\prime}, P_{X}, \times P_{Y}\right)$. But since $H_{1}\left(T^{\prime}, \mathfrak{F}_{X} \times \mathcal{F}_{Y}\right)$ $=L$ is complete, $T^{\prime}$ on $\left(X^{\prime} \times Y, \mathcal{F}_{X}, \times \mathcal{F}_{Y}, P_{X}{ }^{\prime} \times P_{Y}\right)$ is conjugate to a direct product by Theorem 4.5 . 
5. An example. In this section we shall construct an ergodic measure preserving transformation $T$ with quasi-discrete spectrum such that $H_{1}(T)$ can be split: $H_{1}(T)=\widetilde{H}_{1}(T) \times H_{1}^{*}(T)$, but $\tilde{H}_{2}(T)$ is not a direct factor of $H_{2}(T)$. Thus it will follow that $T$ is not conjugate to a direct product as in Theorem 4.5. The proofs of various properties will only be sketched as they are similar to the known algebra constructions (cf. e.g. [5, pp. 202-205]).

Let $T^{\prime}$ be the ergodic measure preserving transformation on $\left(\Omega^{\prime}, \mathcal{F}^{\prime}, P^{\prime}\right)$ with discrete spectrum and with eigenvalues $H_{1}\left(T^{\prime}\right)=\widetilde{K}$ (cf. $[4$, p. 48, Corollary $1]$ ). Let $T$ " be the totally ergodic measure preserving transformation on ( $\Omega$ ", $\left.\mathcal{F}^{\prime \prime}, P^{\prime \prime}\right)$ with quasi-discrete spectrum and with quasi-eigenvalues given by $H\left(T^{\prime \prime}\right)=H_{2}\left(T^{\prime \prime}\right)=\left\{(x, y): x\right.$ and $\left.y \in L_{a}\right\}$ where $a$ is irrational and $L_{a}=$ $\{\exp (2 \pi i q a): q$ is rational\}, with $R(x, y)=(y, 1)$ (cf. $[1, \S 3])$. A complex number $z$ is identified with $(z, 1)$. Let $T=T^{\prime} \times T^{\prime \prime}$ on $(\Omega, \mathcal{F}, P)$. Let $Q$ be a splitting of the exact sequence $1 \rightarrow K \rightarrow G(T) \stackrel{R}{\rightarrow} H(T) \rightarrow 1$. We will view $H\left(T^{\prime}, \mathcal{F}^{\prime}\right)$ and $H\left(T^{\prime \prime}, \mathcal{F}^{\prime \prime}\right)$ as subgroups of $H(T, \mathcal{F})$.

Let $2<p_{1}<p_{2}<\ldots$ where $p_{j}$ are prime numbers and let $r_{j}$ be positive integers. Let $M$ be the subgroup of $H(T, \mathcal{F})$ generated by:

$$
\begin{gathered}
H_{1}\left(T^{\prime \prime}, \mathcal{F}^{\prime \prime}\right)=\left\{(x, 1): x \in L_{\alpha}\right\}, \quad\left\{\exp \left(2 \pi i / p_{j}^{r} j\right): j \geq 1\right\}, \\
\left\{\exp \left(2 \pi i / p_{j}{ }_{j}^{+1}\right)\left(1, \exp \left(2 \pi i / p_{j}\right)\right): j \geq 1\right\} .
\end{gathered}
$$

Let $\varrho$ be the $\sigma$-algebra generated by $Q(M)$. Clearly $R(M) \subseteq M$, and therefore $\varrho$ is invariant under $T$. It can be seen by direct computation that $H_{1}(T, \mathcal{G})=$ $H_{1}(T, \mathcal{G}) \times H_{1}^{*}(T, \mathcal{G})$ where $H_{1}(T, \mathcal{G})$ is generated by $\left\{\exp \left(2 \pi i / p_{j}^{\tau_{j}}\right): j \geq 1\right\}$ and $H_{1}^{*}(T, \mathcal{G})=H_{1}\left(T ", \mathcal{F}^{\prime \prime}\right)$. Also we have $H_{2}(T, \mathcal{G})=\widetilde{H}_{2}(T, \mathcal{G}) \cdot M$. It can be seen that if two does not divide $n$ then $H_{2}\left(Z_{n}\right) \approx H_{1}\left(Z_{n}\right) \times H_{1}\left(Z_{n}\right)$ with $R(x, y)=$ $(y, 1)$. Then it follows that the elements $\exp \left(2 \pi i / p_{j}^{r j}\right) \in \widetilde{H}_{1}^{n}(T, \mathcal{S})$ are of zero height in $\widetilde{H}_{2}(T, \varrho)$. Thus it follows as in $\left[5\right.$, pp. 204-205] that $\widetilde{H}_{2}(T, \mathcal{G})$ is not a direct factor of $H_{2}(T, \mathcal{S})$. Hence $T$ cannot be factored as in Theorem 4.5.

6. Remarks. We mention two of the problems that we have not yet been able to solve.

If two ergodic measure preserving transformations with quasi-discrete spectrum have equivalent systems of quasi-eigenvalues in the sense of Abramov (cf. $[1, \$ 2])$, are they conjugate? The difficulty is that the linear relations in $H$ may not be preserved under the equivalence homomorphism. In the case covered by Theorem 4.6 this does not really matter, but in general we do not know the answer.

Does every factor of an ergodic measure preserving transformation with quasidiscrete spectrum have quasi-discrete spectrum? This is known in the case of totally ergodic transformations (cf. Hahn and Parry [3]). 


\section{BIBLIOGRAPHY}

1. L. Abramov, Metric automorphisms with quasi-discrete spectrum, Izv. Akad. Nauk SSSR Ser. Mat. 26 (1962), 513-530; English transl., Amer. Math. Soc. Transl. (2) 39 (1964), 37-56. MR 26 \#606.

2. F. Hahn and W. Parry, Minimal dynamical systems with quasi-discrete spectrum, J. London Math. Soc. 40 (1965), 309-323. MR 30 \#5292.

3. - Some characteristic properties of dynamical systems with quasi-discrete spectra, Math. Systems Theory 2 (1968), 179-190. MR 37 \#6435.

4. P. Halmos, Lectures on ergodic theory, Publ. Math. Soc. Japan, no. 3, Math. Soc. of Japan, Tokyo, 1956. MR 20 \#3958.

5. A. Kuros, Theory of groups, GITTL, Moscow, 1953; English transl. of 2nd ed., Vol. I, Chelsea, New York, 1955. MR 15, 501; 17, 124.

6. H. Weyl, Uber die Gleichverteilung von Zohlen mod. Eins, Math. Ann. 77 (1916), $313-352$.

DEPARTMENT OF MATHEMATICS, UNIVERSITY OF CALIFORNIA, SANTA BARBARA, CALIFORNIA 93106 